\title{
List of assignments
}

1. red fred

2. red fred with corrections

3. referee's report

4. fire drill

5. FIRE DRILL

6. DENIM FILMS

7. PARKED CARS

8. SAFE AND SOUND

9. TELEVISION PROGRAMMES

10. CUSTOMER COUNT

11. WEEKEND COURSE

12. EASTER TRADINC

13. EASTER TRADING with emboldening and underlining

14. EASTER TRADING with spaced caps

15. SWITCHING ON PROCEDURE

16. VEDASUN HOLIDAYS IN MAJORCA

17. NEW MACHINERY COMMITTEE ACENDA

18. ENVELOPE AND LABEL

19. FAIRHAVEN SQUASH CLUB RULES

20. VEDASEC ORDER FORM

21. VEDASEC ACCOUNTS LETTER

22. MEMO RE S PATEL LTD

23. VEDASEC THERMAL BINDING SERVICE

24. CARD SALES

25. NOTICE TO HOTEL GUESTS

26. URCENT LETTER WITH COPIES

27. WP OPERATOR LETTER

28. MEMO ON QUARTZ WATCHES NOTES

29. THERMAL BINDING PRICE TABLE

30. VEDASEC WORK SHEET TABLE

31. PUBLIC SPEAKINC COURSE NOTICE

32. CALCULATOR EXERCISE A5 PAPER

33. LETTER RE ANNUAL REPORT

34. ANNUAL REPORT CONTENTS PACE

35. MEMO RE THESIS

36. PACE 57 OF THESIS

37. ADVERTISEMENT FOR LOCAL PAPER

38. INVOICE TO RONALD MACINTYRE LTD

39. LETTER TO SUPPLIERS (TOP +2 COPIES)

40. MEMO RE WP SYSTEM 101 AGREEMENT

41. EXTRACT FROM DRAFT AGREEMENT

42. 'THE ADVERTISER' DISTRIBUTION TABLE

43. TYPED PERSONAL LETTER

44. APPLICATION FOR EMPLOYMENT FORM 